\title{
Epidermoid cyst in temple area with skull perforation
}

\author{
Cha Soo Kim, \\ Sung Ho Yoon, \\ Jae Wook Oh, \\ Keun Cheol Lee \\ Department of Plastic and \\ Reconstructive Surgery, Inje University \\ Haeundae Paik Hospital, Inje University \\ College of Medicine, Busan, Korea
}

Epidermoid cysts are benign tumors that account for approximately $1 \%$ of intracranial tumors. In very rare cases, temporally located extradural intradiploic epidermoid cysts can cause neurological symptoms and skull perforation. Herein, we report the case of a 34-year-old woman who underwent successful treatment of an epidermoid cyst in the temporal region accompanied by neurological symptoms. Accurate radiological evaluation and complete removal of the tumor and capsule play a vital role in ensuring favorable long-term outcomes. Computed tomography and magnetic resonance imaging scans can provide an accurate assessment of the extent of intracranial expansion and invasion of the cerebral parenchyma, as well as enabling the precise localization and characterization of the bone defect and mass. In addition, collaborative surgery with a neurosurgeon is required for cases involving intracranial expansion and dural invasion.

Keywords: Dura mater / Epidermal cyst / Skull

\section{INTRODUCTION}

Epidermoid cysts are benign tumors that account for approximately $1 \%$ of intracranial tumors [1]. Epidermoid cysts that develop intracranially can be subdivided into intradural and extradural cysts, with extradural cysts commonly occurring in the intradiploic space. Extradural intradiploic epidermoid cysts can occur in every bone of the craniofacial area, including the frontal and orbital bones [2]. These lesions derive from ectodermal cell remnants within the cranial bones. In rare cases, temporally located extradural intradiploic epidermoid cysts can cause neurological symptoms and skull perforation. When a patient presents with characteristic neurological symptoms, it is important to make a rapid diagnosis through accurate imaging and physical examination, followed by surgical resection, in order to re-

\footnotetext{
Correspondence: Sung Ho Yoon

Department of Plastic and Reconstructive Surgery, Inje University Haeundae Paik Hospital, Inje University College of Medicine, 875 Haeun-daero, Haeundae-gu,

Busan 48108, Korea

E-mail: H00211@paik.ac.kr

Received October 31, 2019 / Revised February 4, 2020 / Accepted February 27, 2020
}

duce the risk of recurrence and complications. Herein, we report the case of a 34-year-old woman who underwent successful treatment of an epidermoid cyst in the temporal region accompanied by neurological symptoms.

\section{CASE REPORT}

A 34-year-old woman with no diseases other than hepatitis B visited our neurosurgical outpatient department with a headache confined to the right side, along with a slowly growing mass in the right temporal area. A computed tomography (CT) scan showed signs of osteoclasia, although the mass had not invaded the brain parenchyma (Fig. 1). At the time, she was advised to monitor the condition over time, and a year later, she visited our plastic surgery department with a worsened headache and a larger mass. In an X-ray examination performed at the department, a lateral radiograph showed a rounded osteolytic lesion on the right anterior part of the skull (Fig. 2). A magnetic resonance imaging (MRI) scan showed a mass measuring approximately $2.4 \times 2.5 \mathrm{~cm}$ that had not invaded the 


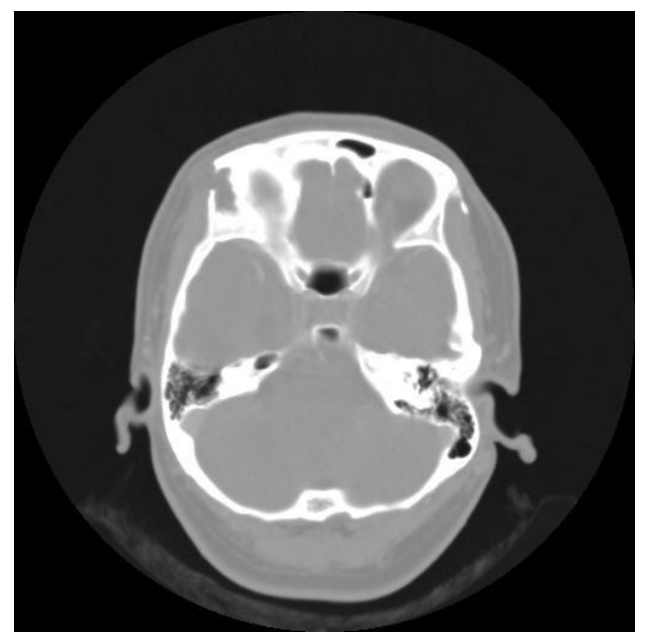

Fig. 1. Computed tomography scan showing well circumscribed, destruction in the right frontal bone.

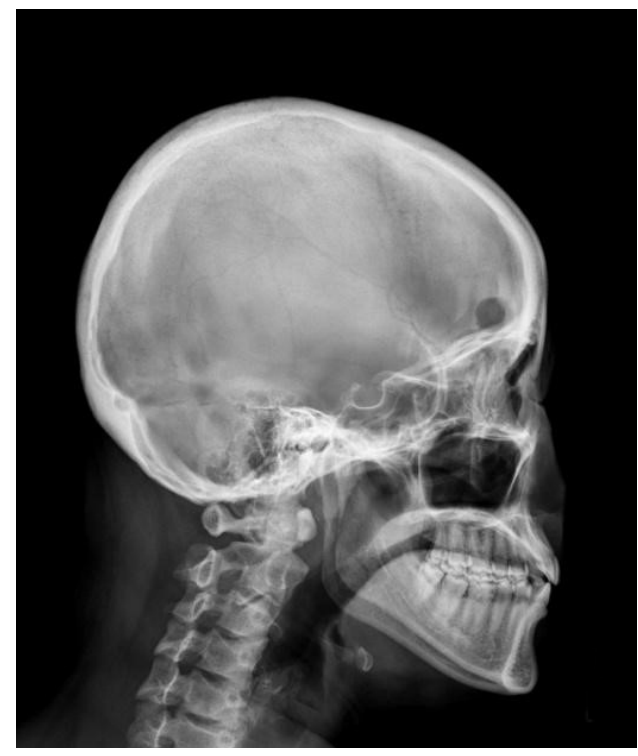

Fig. 2. Plain radiograph showing a roundly marginated lytic defect with scalloped lesion in the right temple area.

brain parenchyma. The mass was hypointense on T1 images and hyperintense on T2 images (Fig. 3). Based on the radiological findings above, epidermoid cyst, dermoid cyst, plexiform neurofibroma, mucocele, and eosinophilic granuloma were considered as differential diagnosis. Most probably the mass was an epidermoid or dermoid cyst $[1,3]$.

Under general anesthesia, an incision was made along the centerline of the mass, and the mass was dissected from the surrounding tissues. The tumor was found to be an encapsulated cystic mass containing a typical soft butter-like semi-solid material, implying an epidermoid cyst. The tumor was completely excised, including the fibrous capsule. The mass was encapsulated and adhered to the lower dura mater, but no dural
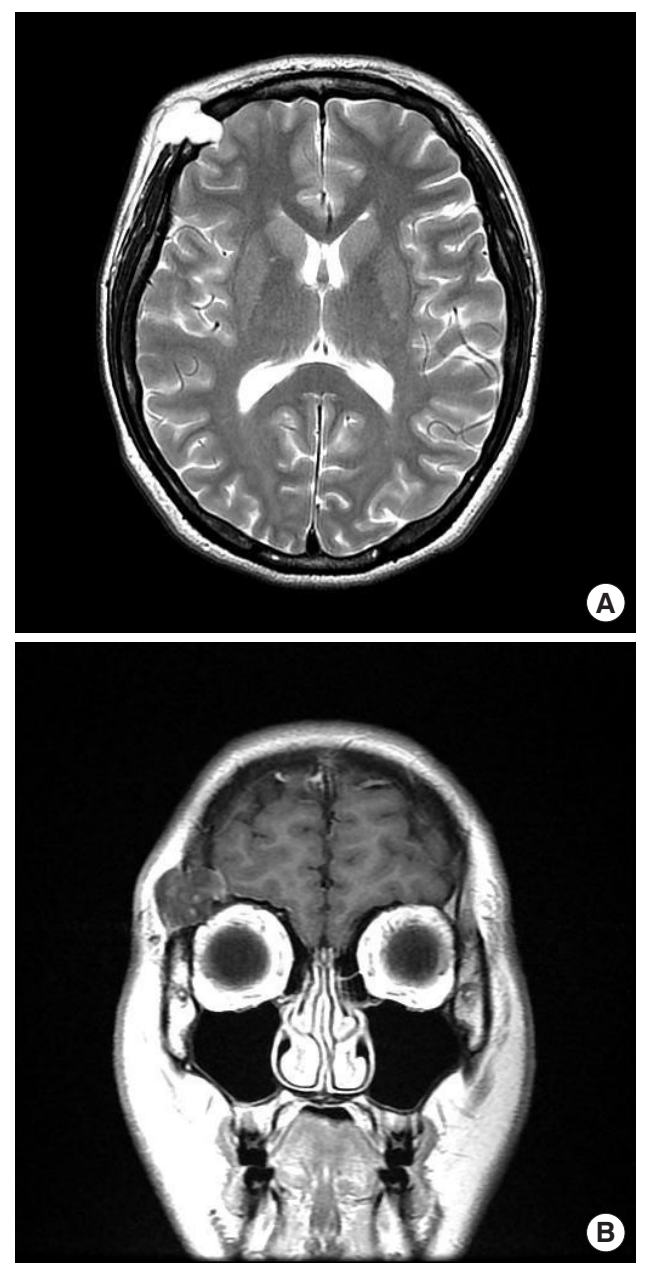

Fig. 3. Magnetic resonance imaging scan. (A) Axial T2-weighted image showing high signal intensity. (B) Coronal T1-weighted image showing low signal intensity.

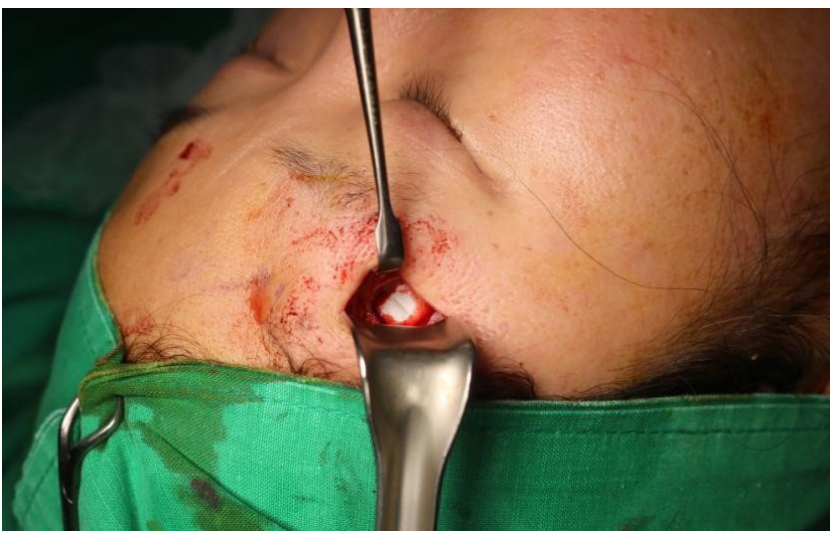

Fig. 4. Intraoperative photograph showing cranioplasty using Medpor Titan (Stryker) after complete surgical resection of the tumor.

invasion was observed. After excision of the tumor, skull reconstruction was performed using Medpor Titan (Stryker, Tuttlingen, Germany) to cover the bone defect (Fig. 4). The patient's neurological symptoms, such as headache, disappeared after 
excision of the mass, and the patient was treated without any postoperative complications. There was no evidence of recurrence at 2 years postoperatively.

\section{DISCUSSION}

Epidermoid cysts originate from ectodermal remnants that remain within the skull bone during embryonic development [4]. The prevalence of extradural epidermoid cyst is very low, accounting for fewer than $0.25 \%$ of all intracranial tumors, and this is the first case report of a mass of this type in the temporal region $[1,2]$. Surgical indications include aesthetic effects, prevention of exacerbation of neurological symptoms, treatment of osteomyelitis, and possible malignant changes in the mass [1]. Accurate radiological evaluation and complete removal of the tumor and capsule are vital for long-term recuperation. For diagnosis, epidermoid cysts should be assessed through imaging studies (radiographs, CT scans, and MRI) and can be confirmed by histological biopsy. As a radiological diagnostic modality, CT enables good assessment of both skull involvement and intracranial extension, as well as facilitating precise localization and characterization of the bone defect and mass. MRI scans can provide an accurate assessment of intracranial expansion and the extent of invasion of the cerebral parenchyma. As in the case described herein, the mass may be associated with extensive destruction of the skull and extracranial extension. In this case, the mass caused skull perforation, but it did not invade the brain parenchyma and the dura mater was not damaged.

Although several previous reports have claimed that epidermoid cysts that undergo repeated surgery and inflammation tend to show malignant transformation, this case was not accompanied by the involvement of surrounding tissues that is seen in malignant tumors [5]. The purpose of surgery in these cases is to carefully dissect the mass from the bone and dura mater to completely remove it, including the capsule, in which case recurrence does not usually occur [6]. To check for dural invasion, collaborative surgery was performed with the neurosurgery department. If dural or cerebral parenchymal invasion is suspected, collaborative surgery is necessary.

Epidermoid cysts can occur anywhere in the intracranial and extracranial spaces. Epidermoid cysts are benign tumors that grow slowly, but if not surgically removed, they can cause inflammation and destroy the surrounding tissue structures [7]. Mass excision is the first-line treatment modality. When the general clinical and imaging findings of extradural intradiploic epidermoid cyst are observed in the cranium, an accurate radiological assessment and complete removal of the tumor and capsule are essential for achieving a favorable long-term prognosis.

We report a rare case of an extradural intradiploic epidermoid cyst located in the temporal region. If a patient has a growing mass in addition to headaches and other neurological symptoms, accurate physical examinations and imaging tests are necessary. In addition, collaborative surgery with a neurosurgeon is required for cases involving intracranial expansion and dural invasion.

\section{NOTES}

\section{Conflict of interest}

No potential conflict of interest relevant to this article was reported.

\section{Ethical approval}

The study was approved by the Institutional Review Board of Inje University Haeundae Paik Hospital (IRB No. 2019-07-049001) and performed in accordance with the principles of the Declaration of Helsinki. Written informed consent was obtained.

\section{Patient consent}

The patient provided written informed consent for the publication and the use of her images.

\section{ORCID}

Cha Soo Kim https://orcid.org/0000-0002-2451-3025

Sung Ho Yoon https://orcid.org/0000-0003-4765-2308

Jae Wook Oh https://orcid.org/0000-0001-8829-0935

Keun Cheol Lee https://orcid.org/0000-0003-0553-0900

\section{RERERENCES}

1. Prall JA, Lloyd GL, Breeze RE. Traumatic brain injury associated with an intradiploic epidermoid cyst: case report. Neurosurgery 1995;37:523-5.

2. Hakyemez B, Aksoy U, Yildiz H, Ergin N. Intracranial epidermoid cysts: diffusion-weighted, FLAIR and conventional MR findings. Eur J Radiol 2005;54:214-20.

3. Suzuki F, Mitsuya K, Handa J. Intradiploeic inclusion cyst lined with upper respiratory epithelium. Acta Neurochir (Wien) 1997; 139:793-4.

4. Toglia JU, Netsky MG, Alexander E Jr. Epithelial (epidermoid) tumors of the cranium: their common nature and pathogenesis. J Neurosurg 1965;23:384-93.

5. Resnick D. Pituitary disorders. In: Resnick D, Niwayama G, 
editors. Diagnosis of bone and joint disorders. Philadelphia: WB Saunders; 1981. p. 1780-1.

6. Rand CW, Reeves DL. Dermoid and epidermoid tumors (cholesteatomas) of the central nervous system: report of 23 cases.
Arch Surg 1943;46:350-76.

7. Hoeffel C, Heldt N, Chelle C, Claudon M, Hoeffel JC. Malignant change in an intradiploic epidermoid cyst. Acta Neurol Belg 1997;97:45-9. 